\title{
A Note on Solvability of the Nonlinear Abstract Viscoelastic Problem in Banach Spaces
}

D. ANDRADE ${ }^{1}$, Departamento de Matemática, Universidade Estadual de Maringá, 87020-900 Maringá-PR, Brazil.

\begin{abstract}
In this work we are concerned with the existence of integral solution for a nonlinear abstract viscoelastic problem in a Banach space where the operator is accretive and time dependent.
\end{abstract}

\section{Introduction}

In this work we are concerned with the solvability of the nonlinear abstract problem in viscoelasticity with memory,

$$
\left\{\begin{array}{l}
\frac{d u}{d t}+A(t) u(t) \ni \int_{0}^{t} k(t-s) g(s, u(s)) d s, 0 \leq t \leq T, \\
u(0)=x \in D=\overline{D(A(0))},
\end{array}\right.
$$

where the family $A(t): D\left((A(t)) \subseteq X \rightarrow 2^{X}, 0 \leq t \leq T\right.$ is at least $m$ - accretives operators in Banach space $X, g:[0, T] \times D \rightarrow X$ and $k:[0, T] \rightarrow L(X)$ are continuous functions, where $L(X)$ is the space of linear operators on $X$. Problems of this type appear in various applied areas and several authors have studied different versions of (1.1). In particular, we note that more general mathematical model of heat conduction in a beam or vibrations of a string both with memory can be rewritten as an integro-differential equation with inclusion. There are another examples as in thermoelastic evolution systems. See [1], [3] and [7].

There are several works and methods on solvability of functional evolution equations with accretive operators dependent on $t$ but as far as we known nothing on viscoelasticity system. The main goal of this work is to prove the existence of integral solution of (1.1). We use the fixed point theorem and auxiliary results to obtain existence of integral solution. The idea of the proof is to define an operator $P$ where its fixed points are integral solutions.

In what follows, by $X$ we denote a real Banach space with norm $\|\cdot\|$ and dual $X^{*}, J$ is the duality mapping. As usual we write

$$
\langle y, x\rangle_{s}=\lim _{h \rightarrow 0^{+}} \frac{\|x+h y\|^{2}-\|x\|^{2}}{2 h},
$$

\footnotetext{
11doherty@dma.uem.br.
} 


$$
\langle y, x\rangle_{i}=\lim _{h \rightarrow 0^{-}} \frac{\|x+h y\|^{2}-\|x\|^{2}}{2 h} .
$$

We know that for every $x, y \in X$ there exists $x_{1}^{*}, x_{2}^{*} \in J x$ such that

$$
\begin{aligned}
& \langle y, x\rangle_{s}=x_{1}^{*}(y)=\sup \left\{x^{*}(y), x^{*} \in J x\right\}, \\
& \langle y, x\rangle_{i}=x_{2}^{*}(y)=\inf \left\{x^{*}(y), x^{*} \in J x\right\} .
\end{aligned}
$$

We note that $\langle,\rangle_{s}$ is upper semicontinuous and $\langle,\rangle_{i}$ is lower semicontinuous. For these facts and additional properties of these directional derivatives the readers are referred to see [1] and [6].

Given a set valued operator $A$ we denote by $D(A)$ and $R(A)$ the sets $\{x \in$ $X, A x \neq \emptyset\}$ and $\cup_{x \in D(A)} A x$, respectively. For simplify set valued operators are identified with their graphs, i.e., if $y \in A x$ we denote by $(x, y) \in A$. An operator $A: D(A) \subseteq X \rightarrow 2^{X}$ is called accretive if only if for every $\lambda \in \mathbb{R}^{+}$and every $y_{1} \in A x_{1}, y_{2} \in A x_{2}$, we have

$$
\left\|x_{1}-x_{2}\right\| \leq\left\|\left(x_{1}-x_{2}\right)+\lambda\left(y_{1}-y_{2}\right)\right\| .
$$

Equivalently, $A$ is accretive if for every $x_{1}, x_{2} \in D(A)$ there exists $j \in J\left(x_{1}-x_{2}\right)$ such that $\left\langle y_{1}-y_{2}, j\right\rangle \geq 0$, for all $y_{1} \in A x_{1}$ and $y_{2} \in A x_{2}$.

An accretive operator is called $m$-accretive if $R(I+\lambda A)=X$, for every $\lambda \in \mathbb{R}^{+}$, here $I$ is the identity mapping.

To introduce the notion of solution of (1.1) we consider the functional problem

$$
\left\{\begin{array}{l}
u^{\prime}(t)+A(t) u(t) \ni f(t), t \in[0, T] \\
u(0)=x_{0}
\end{array}\right.
$$

where $A(t)$ is a set valued nonlinear accretive operator and $f \in C([0, T] ; X)$.

Definition 1.1. We say that $u:[0, T] \rightarrow X$ is a strong solution of $\left(A_{x_{0}}^{f}\right)$ if it is absolutely continuous on compact subsets of $(0, T)$, strongly differentiable on $(0, T)$ and satisfies $\left(A_{x_{0}}^{f}\right)$ for almost every on $(0, T)$.

Next we introduce the so called discrete approximate solution. Let $0=t_{n, 0}<$ $t_{n, 1}<\cdots<t_{n, N(n)}=T$ be a partition of $(0, T)$ such that

$$
\max _{1 \leq k \leq N(n)}\left(t_{n, k}-t_{n, k-1}\right) \rightarrow 0
$$

as $n \rightarrow \infty$. Suppose that there exists $\left\{x_{n, k}\right\} \subset X$ with $x_{n, k} \in D\left(A\left(t_{n, k}\right)\right)$ and

$$
f_{n, k}-\frac{x_{n, k}-x_{n, k-1}}{t_{n, k}-t_{n, k-1}} \in A\left(t_{n, k}\right) x_{n, k}
$$

for every $k=1,2, \ldots, N(n)$, where $x_{n, 0}=x_{0}$ and $f_{n, k}=f\left(t_{n, k}\right)$. 
Definition 1.2. The sequence of step functions $\left\{x_{n, k}\right\}$ defined on $[0, T]$ by

$$
x_{n}(t)= \begin{cases}x_{0}, & t=0, \\ x_{n, k}, & t \in\left(t_{n, k-1}, t_{n, k}\right], \quad k=1,2, \ldots, N(n)\end{cases}
$$

is called a "discrete approximate" solution of $\left(A_{x_{0}}^{f}\right)$. A strongly continuous function $u:[0, T] \rightarrow X$ is called a "weak solution" of $\left(A_{x_{0}}^{f}\right)$ if there exists a sequence of discrete approximate solution of $\left(A_{x_{0}}^{f}\right)$ converging uniformly to $u(t)$ on $[0, T]$ as $n \rightarrow \infty$.

In the rest of this work we consider $A(t), 0 \leq t \leq T$ a family of $m$-accretive operators over the real Banach space $X$ with the following hypotheses.

(H1) For $a<0 \leq s \leq t \leq T<b, \lambda \geq 0$ there exists $h:(a, b) \rightarrow X$ continuous and $L: \mathbb{R}^{+} \rightarrow \mathbb{R}^{+}$take bounded on bounded subsets such that

$$
\left\|x_{1}-x_{2}\right\| \leq\left\|\left(x_{1}-x_{2}\right)+\lambda\left(y_{1}-y_{2}\right)\right\|+\|h(t)-h(s)\| L\left(\left\|x_{2}\right\|\right)
$$

for every $\left(x_{1}, y_{1}\right) \in A(t)$ and $\left(x_{2}, y_{2}\right) \in A(s)$.

(H2) If $t_{n} \uparrow t, x_{n} \in D\left(A\left(t_{n}\right)\right)$ and $x_{n} \rightarrow x$ in $X$, then $x \in \overline{D(A(t))}$.

Now we introduce the notion of integral solution, see [7].

Definition 1.3. By an integral solution on $[s, T]$ for $\left(A_{x_{0}}^{f}\right), x_{0} \in \overline{D(A(s))}$, we mean a continuous function $u:[s, T] \rightarrow X$ such that:

1) $u(s)=x_{0}$

2) $u(t) \in \overline{D(A(t))}$ a.e. $t \in[s, T]$,

3) $\|u(\bar{t})-x\|^{2} \leq 2 \int_{t}^{\bar{t}}\left[\langle f(\tau)-y, u(\tau)-x\rangle_{s}+C\|u(\tau)-x\|\|h(\tau)-h(r)\|\right] d \tau+\|u(t)-x\|^{2}$, where $C=\max \left\{L\left(c_{1}\right), L(\|x\|)\right\}$ and $c_{1}=\sup \{\|u(t)\| ; s \leq t \leq T\}, s \leq t \leq \tau \leq \bar{t} \leq$ $T, r \in[s, T]$ and $(x, y) \in A(r)$.

Hypotheses $\mathbf{H 1}$ and $\mathbf{H 2}$ do not imply that $\overline{D(A(t))}$ is independent of $t$. However we can prove that $m$-accretivity of $A(t)$, together with $\mathbf{H 1}$ and $\mathbf{H} \mathbf{2}$ imply that $\overline{D(A(t))}:=D$ is necessarily independent on $t$. We can prove that if $X$ is uniformly convex then $D(A(t))$ is a convex set, see Barbu [1]. As far as we known to classify Banach the space $X$ such that $\overline{D(A(t))}$ is a convex set is open problem. In the following we suppose that $D$ is convex subset $X$.

The next result due to Pavel is a theorem of existence of integral solution of $\left(A_{x_{0}}^{f}\right)$. The readers are referred to [7] for the proof of the following theorems.

Theorem 1.1. Assume that $A(t), 0 \leq t \leq T$ satisfies $\mathbf{H 1}$ and $\mathbf{H 2}$ and that for each $t \in[0, T] A(t)$ is m-accretive. Moreover, assume $f:[0, T] \rightarrow X$ a continuous function. Then the limit of discrete approximate solutions $u:[0, T] \rightarrow X$ is the unique integral solution of problem $\left(A_{x_{0}}^{f}\right)$.

Moreover, if $v$ is a integral solution of $\left(A_{x_{1}}^{g}\right), x_{1} \in D$, and $g:[0, T] \rightarrow X$ continuous, then

$$
\|u(t)-v(t)\| \leq\|u(s)-v(s)\|+\int_{s}^{t}\|f(\tau)-g(\tau)\| d \tau .
$$


For $u(t)$ and $v(t)$ integral solution of $\left(A_{x_{0}}^{f}\right)$ and $\left(A_{x_{0}}^{g}\right)$ respectively, Kartsatos et al. in $[5]$ proved that

$$
\|u(t)-v(t)\|^{2}-\|u(s)-v(s)\|^{2} \leq \int_{s}^{t}\langle f(\tau)-g(\tau), u(\tau)-v(\tau)\rangle_{s} d \tau .
$$

The organization of the remainder part of this work is as follows. In Section 2 we introduce some basics results. In Section 3 we show the existence of local integral solution to the nonlinear abstract problem and to the problem (1.1).

\section{Auxiliary Results}

Let

$$
D=\{D(t) ; D(t) \subset X, 0 \leq t \leq T\}
$$

be a family of nonempty subsets of the Banach space $X$.

Definition 2.1. A family $U=\{U(t, s), 0 \leq s \leq t \leq T\}$ of operators is said to be a evolution if it satisfies the following conditions with respect to $D$

1) For each pair $(s, t)$ with $0 \leq s \leq t \leq T, U(t, s): D(s) \rightarrow D(t)$ and $U(s, s)=I$ is a identity on $D(s)$.

2) $U(t, s) U(s, r)=U(t, r)$, for $0 \leq r \leq s \leq t \leq T$.

3) For each $s \in[0, T]$ and $x \in D(s)$, the function $t \mapsto U(t, s) x=u(t, s, x)$ is continuous on $[s, T]$.

4) If $0 \leq s_{n} \leq t_{n} \leq T, x_{n} \in D\left(s_{n}\right)$ and $s_{n} \downarrow s, t_{n} \rightarrow t, x_{n} \rightarrow x$ and if $x \in D(s)$, then $U\left(t_{n}, s_{n}\right) x_{n} \rightarrow U(t, s) x$ as $n \rightarrow \infty$.

A condition that implies 4) is the following, see [7]:

5) There exists a real number $w \in \mathbb{R}$ and a function $F$ such that

$$
\|U(t+s, s) x-U(t+r, r) y\| \leq \mathrm{e}^{w t}\|x-y\|+\int_{0}^{t} \mathrm{e}^{w(t-\tau)} F(\tau+s, \tau+r) d \tau,
$$

for $0 \leq r \leq s \leq T, 0 \leq t \leq T-s, x \in D(s), y \in D(r)$ where $F:[0, T] \times[0, T] \rightarrow \mathbb{R}$ is a continuous function with $F(s, s)=0, \forall s \in[0, T]$ and $F(t, s)=F(s, t), F(t, s) \leq$ $F(t, r)+F(s, r), 0 \leq r \leq s \leq t \leq T$.

The "range condition"

$$
\overline{D(A(t))} \subset R(I+h A(t+h)), 0<h<h_{0}, s \leq t \leq T,
$$

for small $h_{0}>0$ together with $\mathbf{H 1}$ and $\mathbf{H 2}$ are sufficient conditions to $A(t)$ generate an evolution operator. If $A(t)$ is a $m$-accretive family, then the range condition is the following

$$
R(I+A(t))=X, t \in[0, T]
$$

which is equivalent to

$$
R(I+h A(t))=X, \forall t \in[0, T], \forall h>0 .
$$


In fact, we can prove, see [7], that hypotheses $\mathbf{H 1}$ and $\mathbf{H 2}$ imply that $A(t)$ generate a evolution operator $U$ in the sense 1)...5) with $D(t)=\overline{D(A(t))}$ and $F(t, s)=$ $C\|h(t)-h(s)\|$, where $C$ is a positive constant that depend of $L$ and of homogeneous solution $\left(A_{x_{0}}^{f}\right)$ with $f=0$. Thus $U(t, s) x_{0}$ is the unique integral solution of $\left(A_{x_{0}}^{f}\right)$ with $f=0$. Now we consider that $U$ is an evolution operator in the sense of 1)..4). The readers are referred to Pavel [7] for the prove of the following theorem.

Theorem 2.1. If $U$ is compact, then for each $0 \leq s<t \leq T, U(t, s)$ is equicontinuous at $t_{0}>s$, on bounded subsets $Y \subset D(s)$.

We can prove that if the evolution operator $U$ generated by a family of $m$-accretive operators $\{A(t), 0 \leq t \leq T\}$ is compact then we have.

I) For each $s<t_{0}, \bar{U}(t, s)$ is an equicontinuous at $t_{0}$ on bounded subsets $Y \subset$ $\overline{D(A(s))}$,

II) For each $s \in[0, T]$ and $\lambda>0, J_{\lambda}=(I+\lambda A(s))^{-1}$ is compact. We remark that an extension of Brezis' Theorem says that the evolution operator $U$ generated by a family of $m$-accretive operators $\{A(t), 0 \leq t \leq T\}$ is compact if and only if conditions I and II hold.

Given $f:[0, T] \rightarrow X$ continuous we denote by $B_{x_{0}} f$ the unique integral solution of $\left(A_{x_{0}}^{f}\right)$. The map $f \mapsto B_{x_{0}}$ is called Bénilan-Pavel operator. From (1.2) we obtain that $B_{x_{0}}$ is a continuous operator on $C([0, T] ; X)$.

\section{Main Result}

We recall that we are assuming that $D$ is a convex subset of $X$. Now we define local integral solution for the following problem

$$
\left\{\begin{array}{l}
\frac{d u}{d t}+A(t) u(t) \ni(F u)(t), 0 \leq t \leq T \\
u(0)=x \in D
\end{array}\right.
$$

where $F: C([0, T] ; D) \rightarrow C([0, T] ; D)$ is a continuous operator.

For $a \in(0, T]$ we introduce $E_{a}$ and $T_{a}$ given by

$$
\begin{aligned}
& E_{a}: C([0, a] ; X) \rightarrow C([0, T] ; X), \\
& T_{a}: C([0, T] ; X) \rightarrow C([0, a] ; X),
\end{aligned}
$$

where

$$
\left(E_{a} u\right)(t)= \begin{cases}u(t), & \text { if } t \in[0, a] \\ u(a), & \text { if } t \in[a, T]\end{cases}
$$

and

$$
\left(T_{a} u\right)(t)=u(t)
$$

It is easy to see that $E_{a}$ and $T_{a}$ are continuous operators. 
Definition 3.1. By a local integral solution of $\left(A_{x}^{F u}\right)$ in $[0, r], 0<r \leq T$ we mean a continuous function $u:[0, r] \rightarrow D$ with $u(0)=x$ a fixed point of $T_{r} B_{x} F E_{r}$. The local solution on $[0, s)$ is a local solution on $[0, r]$ for all $0 \leq r<s$.

The following three theorems are extensions of results contained in [7]. See [2] to details.

Theorem 3.1. Let $A(t), 0 \leq t \leq T$, be a family of $m$-accretive operators on Banach space $X$ satisfying $\mathbf{H 1}$ and $\mathbf{H} \mathbf{2}$ and $x \in D$. Assume further that $F(K)$ is uniformly integrable for every bounded set $K$ and that $B_{x} F$ is a compact operator. Then there exists $\delta>0$ and at least an integral solution of $\left(A_{x}^{F u}\right)$ on $[0, \delta]$.

Let $I(F)=\left\{u ; u\right.$ is a local integral solution on $[0, r]$ of $\left.\left(A_{x}^{F u}\right)\right\}$. As in the proof of case $A(t) \equiv A$ independent of time we can prove the following results using the evolution operator instead of semigroups. See [5], [7] and [4] for the proof in the case independent of $t$.

Theorem 3.2. Let the evolution operator $U(t, s)$ be generated by $A(t), t \in[0, T]$ and let $S \subset C([0, T] ; D)$ be subset such that $F(S)$ is uniformly integrable.

a) If $U(t, s)$ is a compact operator for $s<t$, then the solution set

$$
I(F)=\left\{v ; v \text { is a local integral solution of }\left(A_{x}^{F u}\right) \text { on }[0, r]\right\}
$$

is relatively compact in $C([0, r] ; D)$.

b) If $U(t, s)$ is an equicontinuous then $I(F)$ is a bounded equicontinuous subset of $C([0, r] ; D)$.

Theorem 3.3. Assume that the evolution operator $U(t, s)$ is generated by $A(t), t \in$ $[0, T]$, is a compact operator for $s<t$. Let $S \subset C([0, T] ; D)$ be such that $F(S)$ is uniformly integrable. Then the solution set

$$
I(F(S))=\left\{u_{f} ; u_{f} \text { is an integral solution of }\left(A_{x_{0}}^{f}\right) \text { and } f \in F(S)\right\}
$$

is relatively compact in $C([0, T] ; D)$.

Now we are ready to consider the nonlinear abstract model in viscoelasticity with memory given by (1.1).

Theorem 3.4. Let $A(t), 0 \leq t \leq T$, be a family of $m$-accretive operators satisfying H1 and H2. Suppose that $A(t)$ generate a compact evolution operator $U(t, s), g$ and $k$ as above. Then, for each $x \in D$ there exists $c \in(0, T]$ such that (1.1) have an integral solution in $[0, c]$.

Proof. In fact, let $u_{0}$ be an integral solution of $\left(A_{x}^{f}\right)$ with $f=0$. We take $\rho>$ $0, c_{0} \in(0, T]$ and $M>0$ such that

$$
\begin{gathered}
B(x ; \rho)=\{v \in X:\|v-x\| \leq \rho\} \\
\|g(s, v)\| \leq M,
\end{gathered}
$$


$s \in\left[0, c_{0}\right], v \in D \cap B(x ; \rho)$ and

$$
\left\|u_{0}(t)-x\right\|+c_{0} M=\|U(t, 0) x-x\|+c_{0} M \leq \rho,
$$

$t \in\left[0, c_{0}\right]$, where $\{U(t, s): U(t, s): D \rightarrow D, 0 \leq s \leq t \leq T\}$ is an evolution operator generated by $A(t), 0 \leq t \leq T$.

Let $c_{1} \in\left(0, c_{0}\right]$ and $r \in(0, M]$ such that

$$
c_{1}=\sup _{\tau \in[0, T]}\|k(\tau)\| M \leq r
$$

and

$$
C_{1}=\left\{f \in C\left(\left[0, c_{1}\right] ; D\right):\|f(t)\| \leq r, t \in\left[0, c_{1}\right]\right\} .
$$

We notice that $C_{1}$ is convex, not empty, bounded and closed in $C\left(\left[0, c_{1}\right] ; D\right)$. Since $C_{1}$ is uniformly integrable, it follows from Theorem 3.3. that there exists $c \in\left(0, c_{1}\right]$ such that $B_{x}\left(C_{1}\right)$ is relatively compact in $C([0, c] ; D)$.

Define $C=\{f \in C([0, c] ; D):\|f(t)\| \leq r, t \in[0, c]\}$. If $P: D(P) \subseteq C \rightarrow$ $C([0, c] ; D)$ is given by $\operatorname{Pf}(t)=\int_{0}^{t} k(t-s) g\left(s, u_{f}(s)\right) d s$, where $u_{f}$ is a unique integral solution of $\left(A_{x}^{f}\right)$ and

$$
D(P)=\left\{f \in C: u_{f} \text { is an integral solution of }\left(A_{x}^{f}\right)\right\} .
$$

Note that $P f=f$ implies that $u_{f}$ is an integral solution of (1.1). To complete the proof is sufficient to show that $P$ have a fixed point in $C$.

We affirm that $D(P)=C$, since for any $f \in C$ we have

$$
\begin{aligned}
\left\|u_{f}(t)-x\right\| & \leq\left\|u_{f}(t)-U(t, 0) x\right\|+\|U(t, 0) x-x\| \\
& \leq\|U(t, 0) x-x\|+\int_{0}^{c}\|f(s)\| d s \\
& \leq\|U(t, 0) x-x\|+c r \leq \rho,
\end{aligned}
$$

thus $u_{f}(t) \in D \cap B(x ; \rho)$ and therefore $D(P)=C$.

Note that $P(C) \subset C$, since for any $f \in C$, we have

$$
\begin{aligned}
\|P f(t)\| & \leq \int_{0}^{t}\|k(t-s)\|\left\|g\left(s, u_{f}(s)\right)\right\| d s \\
& \leq c \sup _{\tau \in[0, T]}\|k(\tau)\| M \leq r,
\end{aligned}
$$

for all $t \in[0, c]$. If follows that $P f \in C$.

Moreover the operator $P$ is given by $P=F B_{x}$, where $B_{x}: C \rightarrow C([0, c] ; D)$ is the Bénilan-Pavel operator and $F: C([0, c] ; D) \rightarrow C([0, c] ; D)$ is given by

$$
F u(t)=\int_{0}^{t} k(t-s) g(s, u(s)) d s .
$$


Since $F$ and $B_{x}$ are continuous, then $P$ is continuous from $C$ in itself.

Define $K=\overline{\operatorname{co} P(C)}$. We remark that $K \subseteq C$ and therefore $B_{x}(K)$ is relatively compact set in $C([0, c] ; D)$. Since $F$ is continuous operator we have that $F B_{x}(K)=$ $P(K)$ is a relatively compact in $C([0, c] ; D)$. From Schauder's Theorem $P$ have a fixed point in $K$. That is, there exists $f \in K$ and therefore in $C$ such that $P f=f$. This concludes the proof.

\section{References}

[1] V. Barbu, "Nonlinear semigroups and differential equations in Banach spaces", Nordhoff, Leyden, 1975.

[2] D. Andrade, A note on solvability of the nonlinear abstract viscoelastic problem in Banach Spaces, J.Partial Diff. Equations, 12 (1999), 337-344.

[3] J.S. Gibson, I.G. Rosen and G. Tao, Approximation in Control of Thermoelastic Systems, SIAM J. Control and Optimization, 30 No.5 (1992), 1163-1189.

[4] S. Gutman, Evolutions governed by $m$-accretive plus compact operators, Nonlinear Analysis TMA 7 (1983), 707-715.

[5] A.G. Kartsatos and Ki-Yeon Shin, Solvability of functional evolutions via compactness methods in general Banach Spaces, Nonlinear Analysis TMA, 21 No.7 (1993), 517-535.

[6] V. Lakshmikantham and S. Leela, "Nonlinear differential equations in abstract spaces", Pergamon Press, Oxford, 1981.

[7] N. Pavel, "Nonlinear evolution operators and Semigroups", Lecture Notes in Mathematics, Vol. 1260, Springer Verlag, Berlin, 1987. 